\title{
A Case of Immunoglobulin (Ig) G4-related Sclerosing Periaortitis Mimicking Mediastinal Malignant Tumor
}

\author{
Fuat Erel1, Mustafa Kuzucuoglu22, Nurhan Sarioglu1 and Eren Altun ${ }^{3}$ \\ Department of Pulmonary Medicine1 / Thoracic Surgery2 / Pathology³, Faculty of Medicine, Balikesir University, Balikesir, Turkey
}

\begin{abstract}
Sclerosing mediastinitis was first described by Oulmont in 1855. The aggressive fibro-inflammatory process that occurs in the mediastinum is the end result of a number of inflammatory processes. It is thought that hypersensitivity reactions to various antigens, autoimmune reactions, and idiopathic fibro-inflammatory responses play a role in the etiology of the disease. It has been shown in recent years that immonoglobulin (Ig) G4-related autoimmune diseases may lead to sclerosing mediastinitis. In this study, a rare case of Ig G4-related sclerosing periaortitis is presented in a 50-year male patient. The disease responded well to steroid treatment, but soon recurred following stoppage of the treatment. The treatment with deflazacort was started again; and he responded well. The lesion disappeared within six months of retreatment.
\end{abstract}

Key Words: IgG4, Sclerosing, Periaortitis.

How to cite this article: Erel F, Kuzucuoglu M, Sarioglu N, Altun E. A case of immunoglobulin (ig) g4-related sclerosing periaortitis mimicking mediastinal malignant tumor. J Coll Physicians Surg Pak 2019; 29 (Supplement 2):S144-S147.

\section{INTRODUCTION}

The etiology of sclerosing mediastinitis, which occurs in the mediastinum following an aggressive fibroinflammatory process, is still unknown and is believed to be caused by autoimmune and idiopathic fibroinflammatory processes or from extreme hypersensitivity reactions against antigens of various infectious pathogens. 1

IgG4-related diseases, increasingly recognised in the past 10 years, were shown to have caused sclerosing mediastinitis in many cases. IgG4-related diseases are characterised by aggressive fibrosis and progressive inflammatory processes at any part of the body; and these diseases may mimick infectious and inflammatory disorders and malignancy that can turn into mass formation. ${ }^{2}$

In this study, we present a case which first fully responded, but recurred over a short period of time, showing mass formation around aortic arch. Very few cases have been reported in the literature involving aortic arch and diagnosed as IgG4-related sclerosing periaortitis.

\section{CASE REPORT}

A 50-year male was admitted to chest clinic with the complaint of chronic cough for the last seven years. The

Correspondence to: Dr. Mustafa Kuzucuoglu, Faculty of

Medicine, Department of Thoracic Surgery, Balikesir University

Cagis Kampusu, Balikesir, Turkey

E-mail: mustafakuzucuoglu@hotmail.com

Received: January 08, 2019; Revised: May 25, 2019;

Accepted: June 17, 2019 patient was non-smoker. Postero-anterior chest radiograph of the patient and routine biochemical investigations were normal. No abnormality was detected on physical examination and his respiratory function tests were within normal limits. He was diagnosed as a case of cough-variant asthma, and treated by formetrol and budenoside. For definitive diagnosis, computed tomography (CT) scan chest was done, which showed multiple nodules in the lung parenchyma; largest one measuring at $3 \mathrm{~mm}$, in both lungs, and multiple mediastinal lymph nodules; largest one with $16 \mathrm{~mm}$ diameter, in right upper and lower paratracheal and aorto-pulmonary window.

One year after the follow-up, a thoracic CT scan revealed a new lesion measuring $30 \times 14 \times 12 \mathrm{~mm}$. The new lesion encircled the aorta in the anterior mediastinum. Regression was observed in mediastinal lymph nodes and pulmonary nodules were stable.

In control thorax CT, taken three months later, it was seen that the size of the lesion near the arch of the aorta at the ventral part of brachiocephalic trunk origin increased and reached up to $48 \times 18 \times 16 \mathrm{~mm}$. Meanwhile, no change was detected in the shape, size, and number of pulmonary nodules (Figure 1).

The patient had no clinical complaints during follow-up. Biochemical tests of the patient showed C-reactive protein (CRP) at $9.4 \mathrm{mg} / \mathrm{l}$, erythrocyte sedimentation rate (ESR) of $28 \mathrm{~mm} /$ hour, total $\mathrm{lgE}$ of $9.72 \mathrm{IU} / \mathrm{ml}$, and no peripheral eosinophilia. We performed a skin prick test with inhalant allergens, and the result was negative. While in positron emission tomography CT (PET/CT), SUVmax was 7. Fluorodeoxyglucose (FDG) uptake was 


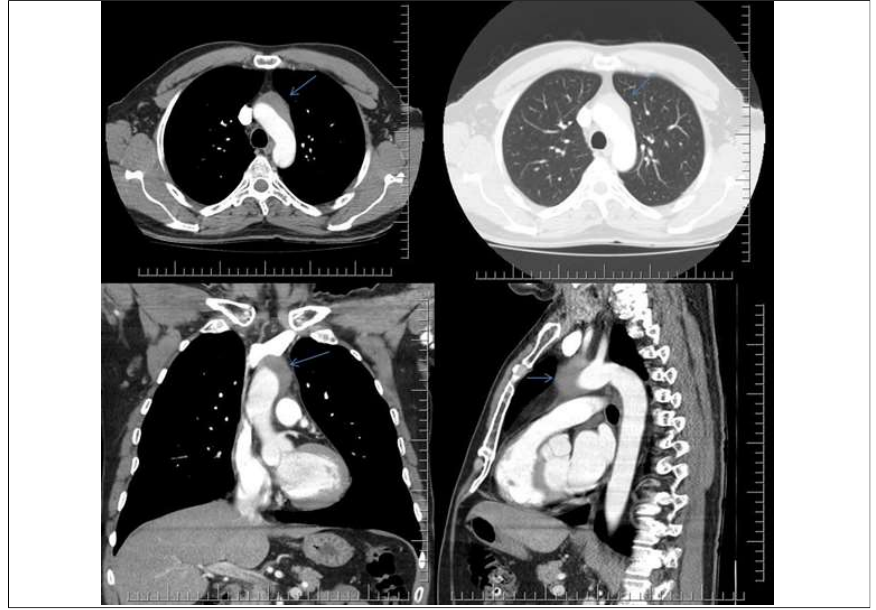

Figure 1: Preoperative computed tomography (CT).

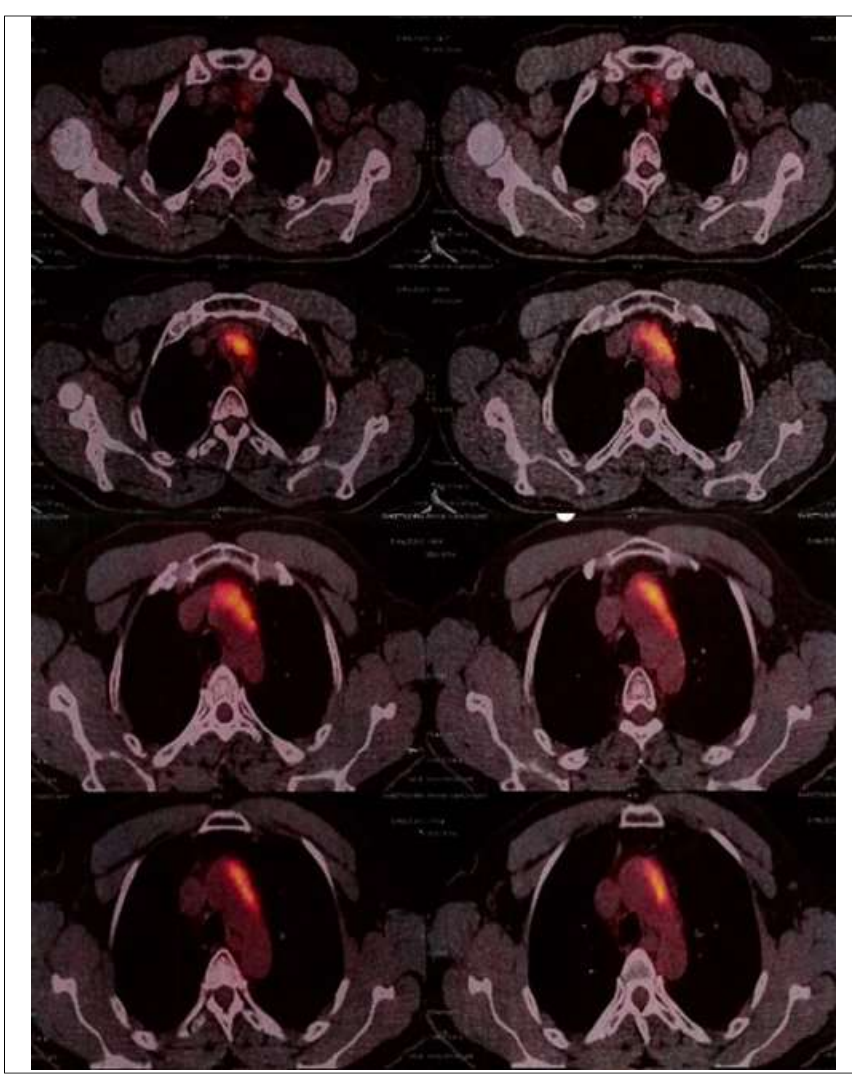

Figure 2: Preoperative positron emission tomography (PET).

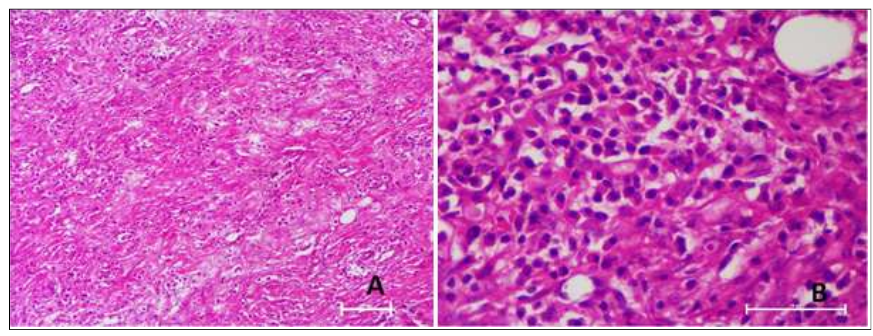

Figure 3: Histopathology of paraaortic mass: (A) Low power view of hematoxylin \& eosine fibrous stroma and mononuclear cells (H\&E X100). (B) High power view of diffuse plasma cell infiltration (H\&E X200).

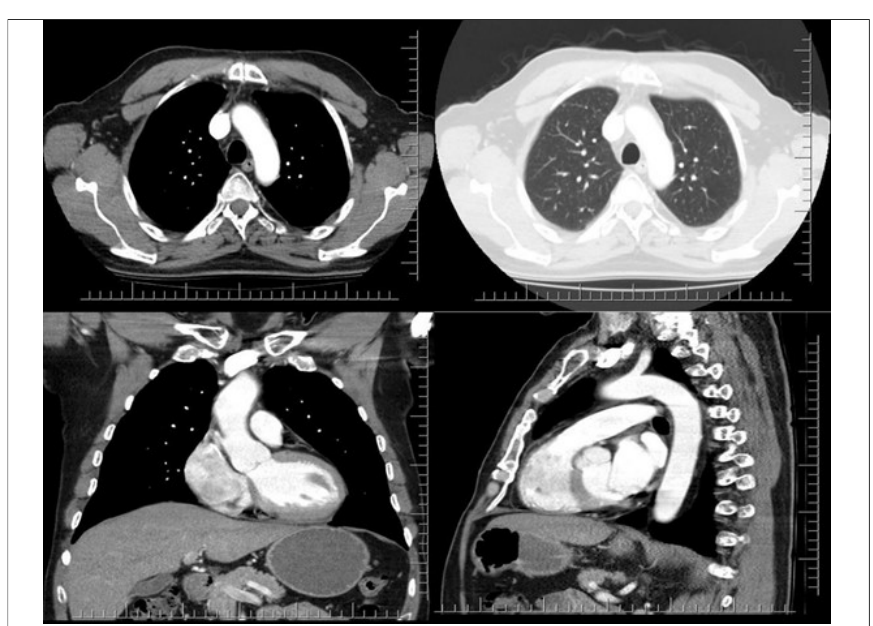

Figure 4: Computed tomography $(\mathrm{CT})$ at the end of third month of treatment.

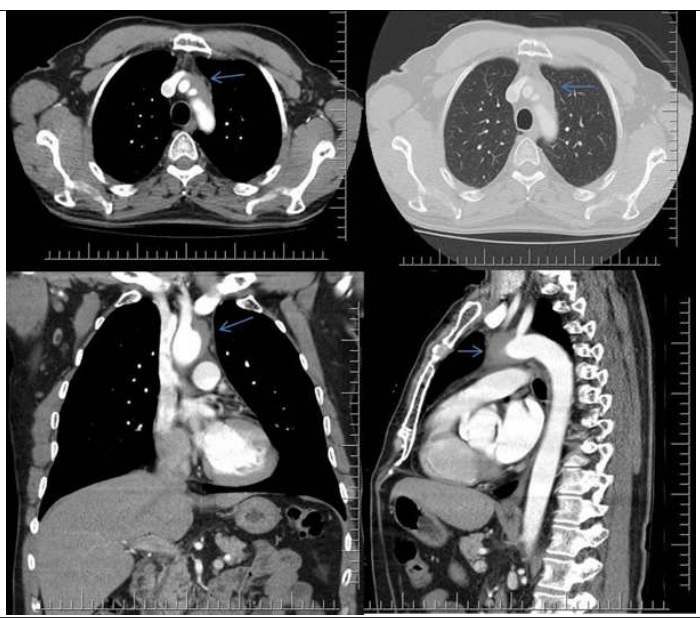

Figure 5: Post-treatment six-month control computed tomography (CT).

observed at the soft tissue mass near aorta, and no FDG uptake was detected in other parts of the body (Figure 2).

With suspected aortic invasion, the patient was directed to chest surgery polyclinic for diagnosis. He did not consent to mediastinoscopy, so diagnostic and therapeutic surgery was planned. During surgery, a mass was detected, which began at the level of proximal end of aortic arch and continued along descending aorta, and wrapping aorta around $120^{\circ}$. As determined by exploration, the mass was invading the aorta and incisional biopsies, taken from the mass, were sent to pathology for frozen study. Since benign-malignant distinction could not be made, the procedure was concluded by taking additional biopsies from the mass. Histopathology report confirmed that it was a case of IgG4-related sclerosing periaortitis (Figure 3).

Serum IgG values were: Total lgG of $1343 \mathrm{mg} / \mathrm{dl}(\mathrm{N}: 700-$ 1600), lgG1 of $942 \mathrm{mg} / \mathrm{dl}$, lgG2 of $341 \mathrm{mg} / \mathrm{dl}$, lgG3 of $40,90 \mathrm{mg} / \mathrm{dl}$, and IgG4 of 19,60 mg/dl (N: 3-201). These values were normal. Treatment was initiated with a daily dose of $90 \mathrm{mg}$ deflazacort. Partial regression was observed 
at the end of the first month, and treatment was continued with $60 \mathrm{mg} /$ day for two more months. At the end of the third postoperative month, significant regression was observed (Figure 4).

Treatment was continued for 6 months with daily dose of 30,15 and $7.5 \mathrm{mg}$ deflazacort; the dose decreased by $50 \%$ each month and the patient was fully recovered. Follow-up CT scan of the patient was done after six months of treatment, which revealed recurrence of the previous disease at same site, which measured $53 \times 34 \times 18 \mathrm{~mm}$ (Figure 5). The CRP and ESR were normal.

Treatment with $60 \mathrm{mg} /$ day deflazacort was started again. Long-term and low-dose treatment was decided. Oneyear treatment was planned with $50 \%$ monthly dose decrease, $6 \mathrm{mg}$ starting from 4th month. Six months after the second treatment, no relapse is observed yet.

\section{DISCUSSION}

Until 1925, it was thought that syphilis and tuberculosis result in sclerosing mediastinitis, which was first recognised in 1855 by Oulmont. Especially, in cases diagnosed in North America, it was revealed that histoplasma capsulatum resulted in mediastinal fibrosis development. ${ }^{1}$ Diseases like fungal infections, tuberculosis or sarcoidosis causing granulomatous reactions, autoimmune diseases and fibro-inflammatory diseases of unknown etiology can be listed as the main causes of sclerosing mediastinitis. ${ }^{3}$

IgG4-related sclerosing mediastinitis can be classified under diseases presenting with autoimmune fibrosis. It shows similarity to autoimmune fibrous diseases in salivary glands, pancreas, bile ducts, and retroperitoneum. Autoimmune pancreatitis was first defined in 1995, and it was shown in 2003 that it is a disease which can present with autoimmune fibrosis in organs other than pancreas. ${ }^{4}$ Hamano et al. detected that the case of sclerosing pancreatitis had high serum IgG4 level; and in immunohistochemical studies, they showed that there were IgG4 positive plasma cells in fibrous tissue. 5

IgG4-related sclerosing diseases are characterised by prominent IgG4-rich lymphoplasmacytic infiltrates, storiform fibrosis and obliterative phlebitis; and high serum IgG4 level is detected in $60-70 \%$ of cases. ${ }^{2-4}$ Pathological diagnosis is the golden standard. ${ }^{2}$

Umehara et al. determined the diagnostic criteria for IgG4-related sclerosing diseases. ${ }^{6}$ According to these criteria, organ dysfunction or nodular lesions and serum lgG4 level $\geq 135 \mathrm{mg} / \mathrm{dl}$ and histopathologically >10 lgG4positive cells or IgG4-positive/lgG-positive cell ratio $\geq 40 \%$ in cross-sectional area, is accepted as a definite IgG4-related sclerosing disease. While organ dysfunction or nodular lesions and histopathologically $>10$ IgG4-positive cell or IgG4-positive/lgG-positive cell ratio $\geq 40 \%$ in cross-sectional area is accepted as probable diagnosis, and organ dysfunction or nodular lesions and serum lgG4 level $\geq 135 \mathrm{mg} / \mathrm{dl}$ as possible diagnosis. According to these criteria, our case was diagnosed as probable IgG4-related sclerosing aortitis with mass appearance around aortic arch at upper mediastinum and detection of lgG4-positive cells in tissue.

Obtaining tissue diagnosis can be difficult due to location of the lesion and may require advanced surgical intervention. Fine needle aspiration biopsies are insufficient, but core biopsies may be beneficial. While Liu et al.4 made diagnosis of mediastinal fibrosis with transthoracic core needle biopsy taken from mass lesion located at posterior mediastinum, Cha et al. ${ }^{7}$ presented the first case of sclerosing periaortitis in the literature that was diagnosed with mediastinoscopy, Oka et al. ${ }^{1}$ could not obtain diagnosis in a female case aged 61 through minimal invasive procedures but could achieve diagnosis by an invasive procedure where the left brachiocephalic vein was taken out through sternotomy.

No standard treatment is available for IgG4-related cases; therefore, the cases should be treated individually. In addition, only symptomatic cases need to be treated. ${ }^{2}$

In some cases, spontaneous remission has been seen. As such, in our case, lymphadenopathies seen in the first tomography can be related to existing disease (with no histopathologic diagnosis), and were cured without any treatment. Since significant tissue damage and fibrosis could develop in some cases, it should be diagnosed at early stages and treated urgently.

Generally, complete response to steroids is seen in the early course of the disease (prednisone $40 \mathrm{mg} /$ day).2,6 The affected organs and the extent of fibrosis are considered to have significant roles in determining the response to immunosuppressive treatments. 7,8

Inoue et al. showed that there was a distinct decline in symptoms and compression of vascular structures with $40 \mathrm{mg} /$ day prednisolone for 4 weeks, and no fibrous tissue development observed throughout the 2-year follow-up. ${ }^{3}$

If treatment by steroids becomes ineffective or appearance of adverse effects precludes its use, rituximab ( $B$ cell depletion therapy) can be utilised. 2

Inoue et al. ${ }^{3}$ suggested that serum IgG4, Ebbo et al. ${ }^{9}$ PET and Wallace et al.10 suggested that plasmablast follow-up is a good indicator for evaluating diagnosis and response to treatment.

In our case, since partial response was achieved in the first month, the treatment was terminated by tapering off the dosage. Serum IgG4 levels were normal at the time of the diagnosis of the disease. Thoracic CT was used to follow-up the treatment response at regular intervals. We observed relapse in thoracic CT, taken at the sixth postoperative month. This indicates that even if the patient does not have complaints, CT follow-up at short intervals is beneficial in early follow-up. 
IgG4-related sclerosing aortitis is an important disease because of its rarity, mimickry of malignancy on imaging, difficulty in differential diagnosis, and difficult to treat.

Since full response can be achieved in IgG4-related sclerosing-aortitis cases by medical treatment, radical surgical approaches with higher mortality and morbidity risks should be avoided, whenever possible.

\section{PATIENT'S CONSENT:}

Informed consent was obtained from the patient to use the descriptive information, radiological images and treatment process.

\section{CONFLICT OF INTEREST:}

Authors declared no conflict of interest.

\section{AUTHORS' CONTRIBUTION:}

FE, MK, NS, EA: Diagnosis, treatment and follow-up of the patient. Written and finally checked the manuscript.

\section{REFERENCES}

1. Oka S, Uramoto H, Yamada S, Tanaka F. Sclerosingmediastinitis of unknown origin: Report of case. Int $J$ Surg Case Rep 2015; 10:5-7.

2. Ataç E, Seyahi E. IgG4 iliskilihastaliklar. TurkiyeKlinikleri J Rheumatol-Special Topics 2014; 7:89-92.
3. Inoue $\mathrm{M}$, Nose $\mathrm{N}$, Nishikawa $\mathrm{H}$, Takahashi $\mathrm{M}$, Zen $\mathrm{Y}$, Kawaguchi M. Successful treatment of sclerosingmediastinitis with a high serum IgG4 level. Gen Thorac Cardiovasc Surg 2007; 55:431-3.

4. Liu X, Yu S, Yu C, He Y, Zhang J, Jiang Y, et al. An IgG4-related sclerosingmediastinitis in posterior mediastinum: $C T$ findings. Radiol Infect Dis 2015; 1:90-3.

5. Hamano H, Kawa S, Horiuchi A, Unno H, Furuya N, Akamatsu T, et al. High serum IgG4 concentrations in patients with sclerosing pancreatitis. N Engl J Med 2001; 344:732-8.

6. Umehara H, Okazaki K, Masaki Y, Kawano M, Yamamoto M, Saeki T, et al. Comprehensive diagnostic criteria for IgG4related disease. Mod Rheumatol 2012; 22:21-30.

7. Cha MJ, Chong S, Kim YS, Park B, Seo JH, Lee ES. Immunoglobulin G4-related periaortitis involving the aortic arch mimicking a mediastinal tumor. Ann Thorac Surg 2017; 103: 267-70.

8. Kamisawa T, Zen Y, Pillai S, Stone JH. IgG4-related disease. Lancet 2015; 385:1460-71.

9. Ebbo M, Grados A, Guedj E, Gobert D, Colavolpe C, Zaidan M, et al. Usefulness of 2-[18F]-fluoro-2-deoxy-D-glucose-positron emission tomography / computed tomography for staging and evaluation of treatment response in IgG4-related disease. Arthritis Care Res 2014; 66:86-96.

10. Wallace ZS, Mattoo H, Carruthers M, Mahajan VS, Della Torre E, Lee $\mathrm{H}$, et al. Plasmablasts as a biomarker for IgG4-related disease, independent of serum IgG4 concentrations. Ann Rheum Dis 2015; 74:190-5. 